\title{
REGULARIZED B1+ MAP ESTIMATION IN MRI
}

\author{
Amanda Funai and Jeffrey A. Fessler \\ EECS Dept., The University of Michigan \\ \{afunai,fessler\}@umich.edu
}

\author{
William Grissom and Douglas C. Noll* \\ BME Dept., The University of Michigan \\ \{wgrissom,dnoll\}@umich.edu
}

\begin{abstract}
A challenge in MR imaging is that RF transmit coils produce non-uniform field strengths, so an excitation pulse will produce tip angles that vary substantially from the desired tip angle over the field of view. For parallel transmit excitation (using a coil array), it is important to have a map of the B1+ field strength (and phase) for RF pulse design. Standard B1+ map estimation methods perform poorly in image regions with low spin density. This paper describes a regularized method for B1+ map estimation using MR scans for each coil and for two or more tip angles. Using these scans and exploiting the fact that maps are generally smooth, the iterative algorithm estimates both the magnitude and phase at each coil's B1+ map. Results from both simulations and real MR data show significant improvements over conventional unregularized methods for $\mathrm{B} 1+$ mapping.
\end{abstract}

Index Terms - Magnetic resonance imaging

\section{INTRODUCTION}

In MRI, a map of the B1+ field strength, called a B1+ map, is essential in many situations. For parallel transmit excitation (using a coil array), e.g., [1], one must have a map of the B1+ field strength (and phase) for RF pulse design. At high fields ( $\geq 3 \mathrm{~T})$, large $\mathrm{B} 1+$ inhomogeneity creates spatially varying signal and contrast; a B1+ map allows for proper pre-scan calibration [2].

A conventional approach to B1+ mapping is to collect two scans, one of which uses twice the RF amplitude of the other, e.g., [2]. Using the double angle formula, a standard methodof-moments estimator is used that ignores noise in the data. This estimator performs poorly in image regions with low spin density.

We propose a new approach that incorporates multiple coils and multiple tip angles as well as accounts for noise in the model. The iterative regularized estimator estimates both the unknown B1+ magnitude and phase map from multiple reconstructed images. The next section first reviews the standard approach for this problem, and then describes our new and improved method with examples of the improved B1+ maps.

\footnotetext{
* Supported in part by NIH grants EB002683 and DA15410.
}

\section{B1+ MAP ESTIMATION}

\subsection{Conventional $\mathrm{B} 1+$ map}

A conventional approach to B1+ mapping is to collect two scans, one of which uses twice the RF amplitude of the other. A model for the reconstructed images is

$$
\begin{aligned}
& y_{j 1}=f_{j} \sin \left(\alpha_{j}\right)+\varepsilon_{1 j} \\
& y_{j 2}=f_{j} \sin \left(2 \alpha_{j}\right)+\varepsilon_{1 j},
\end{aligned}
$$

where $\alpha_{j}$ is the unknown tip angle at the $j$ th voxel. Estimating $\alpha_{j}$ is equivalent to estimating the $\mathrm{B} 1+$ field strength at the $j$ th voxel. Using the double angle formula:

$$
\frac{\mathrm{E}\left[y_{j 2}\right]}{\mathrm{E}\left[y_{j 1}\right]}=\frac{\sin \left(2 \alpha_{j}\right)}{\sin \left(\alpha_{j}\right)}=2 \cos \left(\alpha_{j}\right) .
$$

The standard estimate of $\alpha_{j}$ is a method-of-moments estimator that ignores the noise in the data:

$$
\hat{\alpha}_{j}=\arccos \left(\frac{1}{2} \frac{y_{j 2}}{y_{j 1}}\right) .
$$

This method has several limitations. It performs poorly in image regions with low spin density, i.e., where $y_{j 1}$ is small. It suffers from $2 \pi$ ambiguities if $\alpha_{j}$ is too large, yet it would be sensitive to noise if $\alpha_{j}$ is too small. And it does not immediately generalize to the more general case where we acquire multiple scans to cover a larger range of tip angles, possibly even angles that are larger than $2 \pi$ in some image regions. The estimate (2) also does not provide phase information.

\subsection{Signal model for multiple coils, multiple tip angles}

Suppose there are $K$ coils and we separately transmit from each coil and then receive from a common coil. Suppose for each coil we apply a sequence of $L$ nominal tip angles with known relative RF amplitudes $a_{l}$, for $l=1, \ldots, L$. We model the resulting $K \times L$ reconstructed images as follows:

$$
y_{j k l}=f_{j} \mathrm{e}^{\imath \varphi_{j k}} \sin \left(a_{l} x_{j k}\right)+\varepsilon_{j k l}
$$

for $k=1, \ldots, K$ and $j=1, \ldots, N$, where $f_{j}$ denotes the underlying object transverse magnetization in the $j$ th voxel, 
$\varphi_{j k}$ denotes the phase of the $k$ th coil at the $j$ th voxel, and $\varepsilon_{j k l}$ denotes zero-mean complex gaussian noise. Finally, $x_{j k}$ denotes the unknown "B1+ map" that relates RF amplitude to tip angle at the $j$ th voxel for the $k$ th coil. If the units of the amplitudes $a_{l}$ are gauss, then the units of $x_{j k}$ will be radians per gauss. More typically, the units of $a_{l}$ are arbitrary, and all that is known is their relative values. In this case $x_{j k}$ will have units such that the product of $a_{l}$ and $x_{j k}$ has units of radians. This should suffice for RF pulse design.

The goal is to estimate each B1 magnitude map $\boldsymbol{x}_{k} \triangleq$ $\left(x_{1 k}, \ldots, x_{N k}\right)$ and phase map $\phi_{k} \triangleq\left(\varphi_{1 k}, \ldots, \varphi_{N k}\right)$ from the reconstructed images $\left\{y_{j k l}\right\}$. The underlying magnetization $\boldsymbol{f} \triangleq\left(f_{1}, \ldots, f_{N}\right)$ is also unknown but is a nuisance parameter. We would like the estimator to work robustly even in image regions where $f_{j}$ is small.

If $f_{j}$ were allowed to be complex, then the model above would be non-identifiable because we could add phase to $f$ and subtract the same phase from each $\phi_{k}$ and $\mathrm{E}\left[y_{j k l}\right]$ would remain unchanged. To make the problem identifiable, one could assume that $\varphi_{j 1}$ is zero and then all the other $\varphi_{j k}$ values would be relative phases. Instead, we take the approach of constraining $f$ to be real. This reduces the ambiguity to a sign change of $\boldsymbol{f}$ and a corresponding $\pi$ phase shift in each $\varphi_{j k}$.

Kerr et al. [3] consider a similar problem, except they assume the $a_{l}$ values are powers of two, and they use the following cost function:

$$
\sum_{l}\left(\left|y_{j k l}\right|-\left|f_{j}\right| \sin \left(\left|a_{l} x_{j k}\right|\right)\right)^{2} .
$$

This cost function does not corresponding to the complex gaussian statistical model for the data. They applied a general purpose minimization method from MATLAB. Most importantly, for each voxel they used only the value of tip index $l$ for which the tip was closest to $\pi / 2$. In contrast, we use all the data at every voxel, with a statistically motivated cost function, and a minimization algorithm that is tailored to this problem. We allow arbitrary choices for the $a_{l}$ values, although powers of two may be a reasonable choice.

\subsection{Regularized estimator}

We propose to jointly estimate the B1 magnitude maps $\boldsymbol{x}=$ $\left(\boldsymbol{x}_{1}, \ldots, \boldsymbol{x}_{K}\right)$, the phase maps $\boldsymbol{\phi}=\left(\phi_{1}, \ldots, \phi_{K}\right)$, and the object $\boldsymbol{f}$ by finding minimizers of the following cost function:

$$
\begin{aligned}
\Psi(\boldsymbol{x}, \boldsymbol{\phi}, \boldsymbol{f})= & \sum_{k=1}^{K} \sum_{j=1}^{N} \sum_{l=1}^{L} \frac{1}{2}\left|y_{j k l}-f_{j} \mathrm{e}^{\imath \varphi_{j k}} \sin \left(a_{l} x_{j k}\right)\right|^{2} \\
& +\beta_{1} \mathrm{R}\left(\boldsymbol{x}_{k}\right)+\beta_{2} \mathrm{R}\left(\boldsymbol{\phi}_{k}\right),
\end{aligned}
$$

where $\mathrm{R}\left(\boldsymbol{x}_{k}\right)$ and $\mathrm{R}\left(\phi_{k}\right)$ are regularizing roughness penalty functions, for the magnitude and phase of the B1+ maps, and $\beta_{1}$ and $\beta_{2}$ are regularized parameters that control the smoothness of the estimates.
We use quadratic regularization because B1+ maps are expected be spatially smooth, although edge-preserving regularization could be used if needed. However, we choose not to regularize the magnetization image $f$ because it will contain detailed structural information.

There is no analytical solution for the minimizer of $\Psi$ over all three sets of parameters, so iterative methods are required. We consider an block alternating minimization approach in which we minimize $\Psi$ by cycling over each of the three parameter types and minimizing with respect to one parameter vector while holding the other two at their most recent values.

For given estimates of $\phi$ and $\boldsymbol{x}$, the minimizer of $\Psi$ with respect $f$ is found analytically to be

$$
f_{j}=\operatorname{real}\left(\frac{\sum_{k=1}^{K} \sum_{l=1}^{L} \mathrm{e}^{-\imath \varphi_{j k}} \sin \left(a_{l} x_{j k}\right) y_{j k l}}{\sum_{k=1}^{K} \sum_{l=1}^{L} \sin ^{2}\left(a_{l} x_{j k}\right)}\right) .
$$

For given $\phi$ and $\boldsymbol{f}$ values, the problem of minimizing $\Psi$ with respect to the B1 map $\boldsymbol{x}_{k}$ appears nontrivial because of the nonlinearity of $\sin \left(a_{l} x_{j k}\right)$. Consider just one term in this cost function:

$$
\begin{aligned}
\psi(y, g, a, x) & =\frac{1}{2}|y-g \sin (a x)|^{2} \\
& \equiv-\operatorname{real}\left(y g^{*}\right) \sin (a x)+\frac{1}{2}|g|^{2} \sin ^{2}(a x)
\end{aligned}
$$

The relevant derivatives of this term are:

$$
\begin{aligned}
\frac{\partial}{\partial x} \psi & =-\operatorname{real}\left(y g^{*}\right) a \cos (a x)+|g|^{2} a \sin (a x) \cos (a x) \\
& =-a \cos (a x) \operatorname{real}\left(g^{*}(y-g \sin (a x))\right) \\
\frac{\partial^{2}}{\partial x^{2}} \psi & =\operatorname{real}\left(y g^{*}\right) a^{2} \sin (a x)+|g|^{2} a^{2} \cos (2 a x) .
\end{aligned}
$$

An upper bound for the curvature is

$$
\begin{aligned}
\frac{\partial^{2}}{\partial x^{2}} \psi(y, g, a, x) & \leq a^{2}\left[|y g| \sin (a x)+|g|^{2} \cos (2 a x)\right] \\
& \approx a^{2}|g|^{2}\left[\sin ^{2}(a x)+\cos (2 a x)\right] \\
& =a^{2}|g|^{2} \cos ^{2}(a x) \leq a^{2}|g|^{2} .
\end{aligned}
$$

Thus, using quadratic majorizer principles [4], a natural iteration for updating $\boldsymbol{x}_{k}$ is:

$$
\boldsymbol{x}_{k}^{(n+1)}=\boldsymbol{x}_{k}^{(n)}-\operatorname{diag}\left\{\frac{1}{b_{j}}\right\} \nabla_{\boldsymbol{x}_{k}} \Psi\left(\boldsymbol{x}^{(n)}, \boldsymbol{\phi}, \boldsymbol{f}\right),
$$

where

$$
b_{j} \triangleq \sum_{l=1}^{L} a_{l}^{2}\left|f_{j}\right|^{2}+r \beta_{1} .
$$

The factor " $r$ " depends on the choice of the regularizer $\mathrm{R}\left(\boldsymbol{x}_{k}\right)$. For 2nd-order finite differences with the 8 nearest neighbors, this factor is $4 \cdot 4 \cdot(2+2 / \sqrt{2})$.

For given $\boldsymbol{f}$ and $\boldsymbol{x}$ values, the problem of finding the minimizer with respect to the phase map $\phi$ has essentially the 
same mathematical form as the fieldmap estimation problem described in [5]. In particular,

$$
\begin{array}{r}
\sum_{k=1}^{K} \sum_{l=1}^{L} \frac{1}{2}\left|y_{j k l}-f_{j} \mathrm{e}^{\imath \varphi_{j k}} \sin \left(a_{l} x_{j k}\right)\right|^{2} \\
\equiv-\sum_{k=1}^{K} \operatorname{real}\left(\left[f_{j} \sum_{l=1}^{L} \sin \left(a_{l} x_{j k}\right) y_{j k l}\right] \mathrm{e}^{-\imath \varphi_{j k}}\right) .
\end{array}
$$

Therefore we can apply a similar iteration as described in [5] with appropriate variables, as follows:

$$
\phi_{k}^{(n+1)}=\phi_{k}^{(n)}-\operatorname{diag}\left\{\frac{1}{d_{j}}\right\} \nabla_{\phi_{k}} \Psi\left(\boldsymbol{x}, \phi^{(n)}, \boldsymbol{f}\right),
$$

where

$$
d_{j} \triangleq \sum_{l=1}^{L}\left|y_{j k l} f_{j} \sin \left(a_{l} x_{j k}\right)\right|+r \beta_{2} .
$$

Note that the only "coupling" between the estimates for the various coils occurs in the object update (5). The updates to the B1+ magnitude and phase maps are decoupled across coils, so they could be parallelized.

The cost function $\Psi$ is nonconvex, so the alternating minimization algorithm described above will descend from the initial estimates to a local minimum [6]. . Thus it is desirable to choose reasonable initial estimates. For $\boldsymbol{x}_{k}^{(0)}$, the standard double angle method (2) is a natural choice. For initializing the phasemap, (7) suggests:

$$
\varphi_{j k}^{(0)}=\angle\left(f_{j} \sum_{l=1}^{L} \sin \left(a_{l} x_{j k}\right) y_{j k l}\right) .
$$

Finally, for $\boldsymbol{f}^{(0)}$ we use (5).

\subsection{Simulation Study}

To evaluate the regularized B1+ map estimation method described above, we performed a simulation study using the synthetic true maps shown in Fig. 1. We simulated noisy reconstructed images for $L=3$ different nominal tip angles and $K=4$ different transmit coils using the measurement model (3). The relative amplitudes were $a_{l}=(10,20,30)$. We added complex gaussian noise such that the SNR, defined by $10 \log _{10}(\|\boldsymbol{y}\| /\|\boldsymbol{y}-\mathrm{E}[\boldsymbol{y}]\|)$ was about $21 \mathrm{~dB}$, yielding the images shown in Fig. 2. Fig. 3 shows the initial estimates using the methods described. Note that the standard double angle method only uses two of the three scans. Fig. 4 shows the regularized estimates.

The reduced noise due to regularization and due to using all the scan data is evident. Fig. 3 shows the conventional estimate for the B1 map. Not only is this image very noisy, but there the B1 map is not properly estimated in the large signal void of the skull. The proposed method smoothly interpolates across this signal void for a smooth B1 map in the region of interest as seen in Fig. 4. Similarly, signal voids can be seen in the initial estimate of the phase map yet are smoothed appropriately in the final estimate.

We calculated the error of both the conventional and our new estimate for all four coils. We used a mask to include only those points inside the object (not the background). The combined RMSE for the four coils was .058 for the conventional estimate, and .020 for the new estimate, a reduction of more than half. This clearly shows the effect of less noise and interpolating across the signal voids. Similarly, the final estimate for the B1+ phase map has a RMSE of only .11 while the initial estimate has an RMSE of .86 - almost eight times greater.

We also used this algorithm on real MR data. We used a phantom with coils positioned to create a B1 map that was much larger on one side than on the other. We obtained images at eighteen tip angles from 10 degrees to 180 degrees. Fig. 5 shows the results from the conventional estimate (with tips at $30^{\circ}$ and $60^{\circ}$ ) (2) as well as using the proposed regularized estimator with three of the tip angles $\left(30^{\circ}, 60^{\circ}, 90^{\circ}\right)$ and with all eighteen. The regularized estimates are much smoother than the conventional estimate. This matches our supposition that the phantom should have a smooth B1 map. We see that even using just three images produces a much smoother image than the conventional estimate.

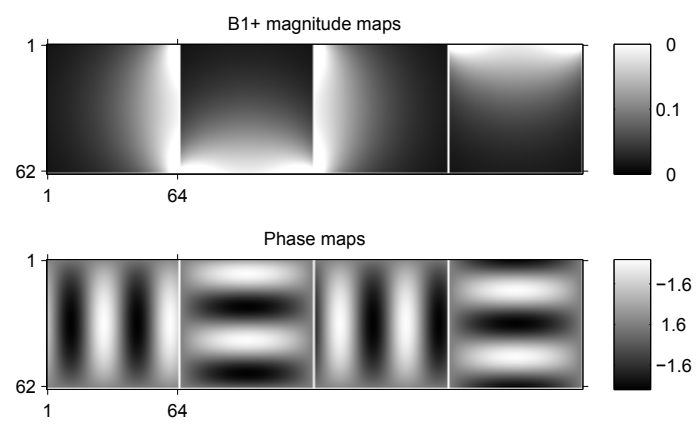

Fig. 1. True B1+ magnitude and phase maps used in simulation.

\section{DISCUSSION}

We have described a new regularized method for B1+ mapping which estimates both the B1 magnitude and phase. This method allows for multiple coils allowing for easy use in designing pulse sequences for parallel excitation. This method yields $\mathrm{B} 1+$ maps that interpolate smoothly over regions with low spin density. This avoids noisy estimates in these regions as well as $2 \pi$ ambiguities that plague the conventional estimate. The conventional estimate uses only two tip angles, while our method allows for any arbitrary selection of angles. 


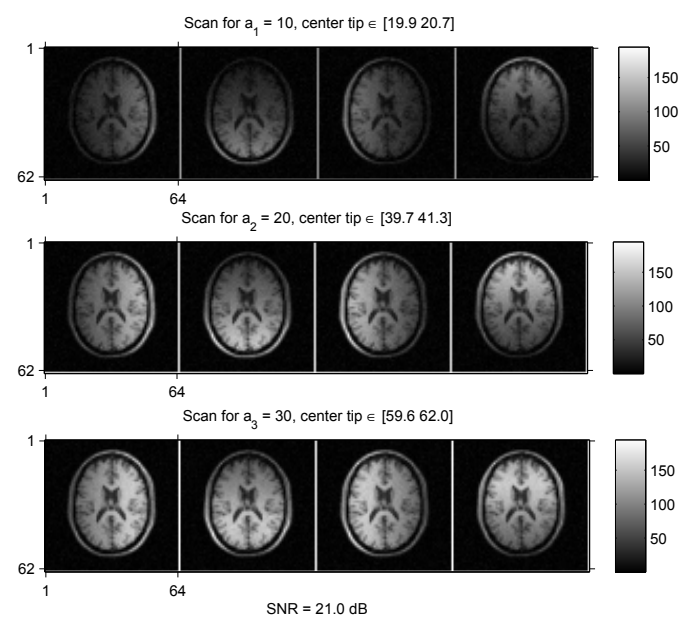

Fig. 2. Simulated MR scans for $L=3$ different nominal tip angles for $K=4$ different transmit coils.

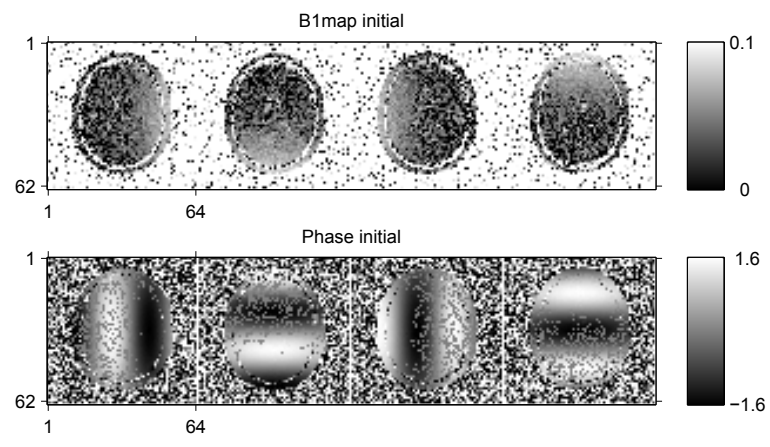

Fig. 3. Initial estimates of B1 maps (2).

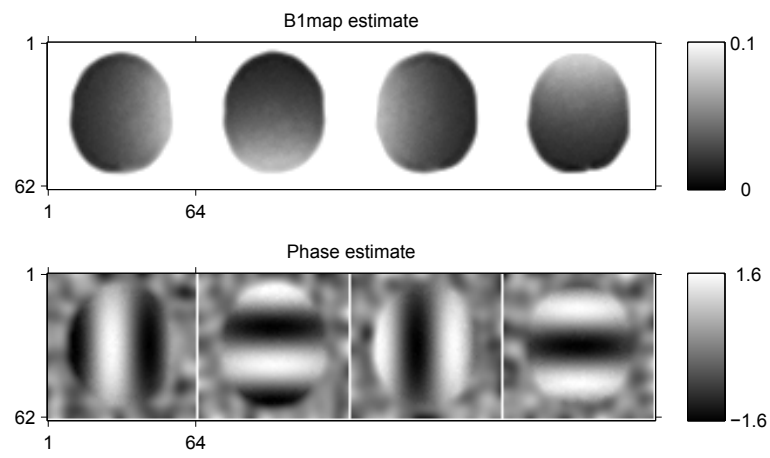

Fig. 4. Regularized estimates of B1 maps.
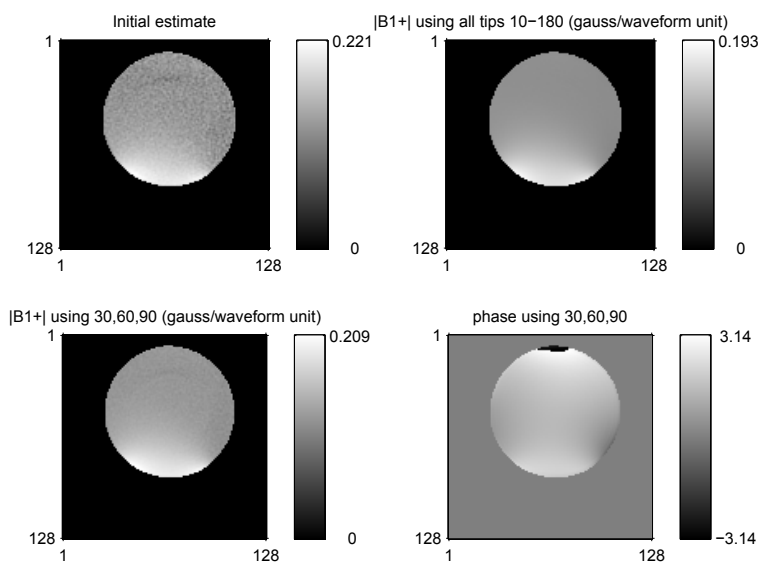

Fig. 5. Top: conventional estimate of B1 using two images; regularized estimate of $\mathrm{B} 1$ using all eighteen images; Bottom: regularized estimate of $\mathrm{B} 1$ using three images; regularized estimate of the phase map.

The simulation results show a sharp decrease in the amount of noise as well as interpolating across areas with low spin density compared to the conventional estimate. The RMSE of the new B1 map is less than half that of the conventional estimate. These gains make this an appropriate method even when using only one coil and the standard two angles.

\section{REFERENCES}

[1] U. Katscher, P. Brnert, C. Leussler, and J. S. van den Brink, "Transmit SENSE," Mag. Res. Med., vol. 49, no. 1, pp. 144-50, Jan. 2003.

[2] C. H. Cunningham, J. M. Pauly, and K. S. Nayak, "Saturated double-angle method for rapid $B_{1}+$ mapping," Mag. Res. Med., vol. 55, no. 6, pp. 1326-1333, June 2006.

[3] A. B. Kerr, C. H. Cunningham, J. M. Pauly, R. O. Giaquinto, R. D. Watkins, and Y. Zhu, "Self-calibrated transmit SENSE," in Proc. Intl. Soc. Mag. Res. Med., 2006, p. 2561.

[4] D. Böhning and B. G. Lindsay, "Monotonicity of quadratic approximation algorithms," Ann. Inst. Stat. Math., vol. 40, no. 4, pp. 641-63, 1988.

[5] J. A. Fessler, D. Yeo, and D. C. Noll, "Regularized fieldmap estimation in MRI," in Proc. IEEE Intl. Symp. Biomed. Imag., 2006, pp. 706-9.

[6] M. W. Jacobson and J. A. Fessler, "An expanded theoretical treatment of iteration-dependent majorize-minimize algorithms," IEEE Trans. Im. Proc., 2006, Submitted. 